BULL. AUSTRAL. MATH. SOC.

VOL. 27 (1983), 269-283.

\title{
ON A FUNCTIONAL DIFFERENTIAL EQUATION IN LOCALLY CONVEX SPACES
}

\author{
SADAYUKI YAMAMURO
}

\begin{abstract}
The notion of accretiveness for multi-valued nonlinear maps is defined in locally convex spaces and it is used to obtain a locally convex space version of a result of M.G. Crandall and J.A. Nohel.
\end{abstract}

\section{0 . Introduction}

The aim of this note is to obtain a locally convex version of a result of Crandall and Nohel [2] about the existence of a unique solution of an initial value problem, where the functions involved have their values in a Banach space. The differential equation in the problem contains a multivalued map on this Banach space. We shall replace this Banach space with a class of locally convex spaces. To carry out this project, we shall use a method which has been introduced in [6] and developed in [7] and [8]. We begin with a brief account of this method.

\section{1. $\Gamma$-completions of locally convex spaces}

Let $E$ be a vector space and $p$ be a semi-norm of $E$. A sequence $\left(x_{i}\right)$ in $E$ is said to be $p$-cauchy if $p\left(x_{i}-x_{j}\right) \rightarrow 0$ as $i, j \rightarrow \infty$. Two p-Cauchy sequences $\left(x_{i}\right)$ and $\left(y_{i}\right)$ are said to be equivalent if $p\left(x_{i}-y_{i}\right) \rightarrow 0$ as $i \rightarrow \infty$.

Received 19 November 1982. This work was completed while the author stayed at the Mathematics Research Center, University of Wisconsin. The author expresses gratitude for their hospitality. 
Let $\left(x_{i}\right)$ be a $p$-Cauchy sequence and $x$ be the set of all $p$-Cauchy sequences in $E$ which are equivalent to $\left(x_{i}\right)$. Such a set $\underline{x}$ is called a $p$-class on $E$. The set of all p-classes on $E$ will be denoted by $E[p]$ and it will be called the p-completion of $E$. It is a vector space when $\alpha \underline{\underline{x}}+\beta \underline{\underline{y}}$ is defined to be the $p$-class which contains the sequence $\left(\alpha x_{i}+B y_{i}\right)$ for some $\left(x_{i}\right) \in \underline{\underline{x}}$ and $\left(y_{i}\right) \in \underline{\mathrm{y}}$. The zero element of $E[p]$ is, therefore, the p-class which contains a p-null sequence.

For $\underline{\underline{x}} \in E[F]$, we define

$$
p(\underline{\underline{x}})=\lim _{i \rightarrow \infty} p\left(x_{i}\right) \text { for }\left(x_{i}\right) \in \underline{\underline{x}} .
$$

Then the value $p(\underline{\underline{x}})$ does not depend on the choice of $\left(x_{i}\right)$ from $\underline{\underline{x}}$. It is obvious that $p$ is a norm on $E[p]$ and, with this norm, $E[p]$ is a Banach space.

For each $x \in E$, let $S_{p}(x)$ be the element of $E[p]$ which contains the $p$-Cauchy sequence whose terms are identical to $x$. Then we have

$$
p\left(S_{p}(x)\right)=p(x) \text { for every } x \in E \text {. }
$$

For $\underline{\underline{x}} \in E[p]$ and $\left(x_{i}\right) \in \underline{\underline{x}}$, we have

$$
\lim _{i \rightarrow \infty} p\left(S_{p}\left(x_{i}\right)-\underline{\underline{x}}\right)=0 \text {, }
$$

which shows that $S_{p}(E)$ is a dense subset of $E[p]$.

Let $E$ be a locally convex space. A directed set $\Gamma$ of semi-norms on $E$ which induces the topology of $E$ will be called a calibration for $E$. Then, for each $p \in \Gamma$, we have the $p$-completion $E[p]$ of $E$. The family $\{E[p]: p \in \Gamma\}$ of Banach spaces will be called the $\Gamma$-completion of $E$. Thus we have a projective system

$$
S_{p}: E \rightarrow E[p] \text { for all } p \in \Gamma \text {. }
$$

It is easy to see that the projective topology on $E$ defined by this system coincides with the topology of $E$.

When $q \geq p$ in $\Gamma$, that is, $q(x) \geq p(x)$ for every $x \in E$, we have the natural embedding 


$$
T_{q, p}: E[q] \rightarrow E[p],
$$

which maps every $\underline{\underline{x}} \in E[q]$ to the $p$-class which contains elements of $\underline{\underline{x}}$. Obviously, this map is linear,

$$
p\left(T_{q, p}(\underline{\underline{x}})\right) \leq q(\underline{\underline{x}}) \text { for every } \underline{\underline{x}} \in E[q]
$$

and

$$
T_{q, p} \circ S_{q}=S_{p}
$$

Furthermore, it is evident that $T_{q, p}(E[q])$ is a dense subset of $E[p]$.

The following fact will be used frequently. For the proof, we refer to $[3]$, p. 231 .

(1.1). Let $E$ be a locally convex space and $\Gamma$ be a calibration for $E$. Then $E$ is complete if and only if the following condition is satisfied: if $\underset{\mathrm{x}}{\Rightarrow} \in E[p]$ for all $p \in \Gamma$ and

$$
T_{q, p}\left(\underline{\mathrm{x}}_{q}\right)=\stackrel{\mathrm{x}}{=} \text { whenever } q \geq p \text { in } \Gamma \text {, }
$$

then there exists $x \in E$ such that $S_{p}(x)=\underline{x}_{p}$ for all $p \in \Gamma$.

\section{2. $\Gamma$-extensions of multi-valued maps}

Let $E$ and $F$ be locally convex spaces and let $\Gamma$ be a calibration for $(E, F)$. In other words, each $p \in \Gamma$ has the $E$-component $p_{E}$ and the $F$-component $p_{F}$ and

$$
\Gamma_{E}=\left\{p_{E}: p \in \Gamma\right\} \text { and } \Gamma_{F}=\left\{p_{F}: p \in \Gamma\right\}
$$

are calibrations for $E$ and $F$ respectively. We shall denote the embeddings $S_{p_{E}}$ and $S_{p_{F}}$ by the same $S_{p}$.

Let $A$ be a multi-valued map of $E$ into $F$, that is, $A$ is a subset of the product $E \times E$. For $p \in \Gamma$ and $[x, y] \in A$, we set

$$
s_{p}([x, y])=\left[S_{p}(x), S_{p}(y)\right] \text {. }
$$

Then

$$
S_{p}(A) \subset E[p] \times F[p],
$$


and we set

$$
A_{p}=\overline{S_{p}(A)}
$$

where the closure is taken in the product $E[p] \times F[p]$ of Banach spaces $E[p]$ and $E[p]$. Hence $A_{p}$ is always closed and it is easy to see that $A_{p}=(\bar{A})_{p}$.

(2.1). $\bar{A}=\bigcap_{p \in \Gamma} S_{p}^{-1}\left(A_{p}\right)$.

Proof. Since $S_{p}(A) \subset A_{p}$, we have

$$
S_{p}(\bar{A}) \subset \overline{S_{p}(A)}=A_{p} \text { for all } p \in \Gamma
$$

To prove the converse, assume that there exists $[x, y] \in S_{p}^{-1}\left(A_{p}\right)$ for all $p \in \Gamma$ such that $[x, y] \vDash \vec{A}$. Then, since $\Gamma$ is directed, there exist $p \in \Gamma$ and $\alpha>0$ such that

$$
\left([x, y]+U_{E}(p, \alpha) \times U_{F}(p, \alpha)\right) \cap A=\emptyset
$$

where $U_{E}(p, \alpha)$ and $U_{F}(p, \alpha)$ are open $p$-balls around zeros with radius $\alpha$ in the spaces $E$ and $F$ respectively. However, for this $p$, since $S_{p}([x, y]) \in A_{p}$, we can choose $\left[x_{i}, y_{i}\right] \in A$ such that $S_{p}\left(\left[x_{i}, y_{i}\right]\right) \rightarrow S_{p}([x, y])$, which is a contradiction.

As usual, the domain of $A$ is denoted by $D(A)$.

(2.2). (i) $\overline{D(A)}=\bigcap_{p \in \Gamma} S_{p}^{-1}\left(\overline{D\left(A_{p}\right)}\right)$

(ii) $\overline{D\left(A_{p}\right)}=\overline{S_{p}(\overline{D(A))}}$.

Proof. Let $x \in \overline{D(A)}$ and choose a net $\left(x_{\lambda}\right)$ in $D(A)$ such that $x_{\lambda} \rightarrow x$. Then $S_{p}\left(x_{\lambda}\right) \in D\left(A_{p}\right)$ and $S_{p}\left(x_{\lambda}\right) \rightarrow S_{p}(x)$. Hence $S_{p}(x) \in \overline{D\left(A_{p}\right)}$, which holds for every $p \in \Gamma$. Conversely, assume that $S_{p}(x) \in \overline{D\left(A_{p}\right)}$ for every $p \in \Gamma$ and $x \notin \overline{D(A)}$. We choose $p \in \Gamma$ and $\alpha>0$ such that

$$
\left(x+U_{E}(p, \alpha)\right) \cap D(A)=\emptyset
$$


For this $p$, since $S_{p}(x) \in \bar{D}\left(\hat{A}_{p}\right]$, we can find $x_{n} \in D\left(A_{p}\right)$ such that $\underline{x}_{n} \rightarrow S_{p}(x)$. Since there exist $x_{n} \in D(A)$ such that

$$
p\left(S_{p}\left(x_{n}\right)-\underline{x}_{n}\right)<1 / n
$$

we can conclude that $s_{p}\left(x_{n}\right) \rightarrow S_{p}(x)$, which is a contradiction. Thus $(i)$ was proved. The proof of $(i i)$ is similar.

(2.3). Assume that $q \geq p$ in $\Gamma$. Then, for every $\stackrel{\mathrm{x}}{q}_{q} \in D\left(A_{q}\right)$,

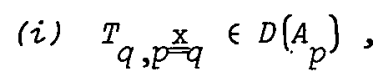

(ii) $T_{q, p}{ }_{q} \stackrel{x_{q}}{=} \subset A_{p}{ }_{q, p} \stackrel{x}{=}$.

Proof. For $\underline{x}_{q} \in D\left(A_{q}\right)$, assume that $\left[\underline{x}_{q}, \underline{x}_{q}\right] \in A_{q}$ and choose $\left[x_{i}, y_{i}\right] \in A$ such that $s_{q}\left(\left[x_{i}, y_{i}\right]\right) \rightarrow\left[\underline{x}_{q}, \underline{x}_{q}\right]$. Then

$$
S_{p}\left(\left[x_{i}, y_{i}\right]\right)=T_{q, p} \circ S_{q}\left(\left[x_{i}, y_{i}\right]\right) \rightarrow T_{q, p}\left[\underline{x}_{q}, \underline{y}_{q}\right] \text {, }
$$

where we used the following notation:

$$
T_{q, p}\left[\underline{\mathrm{x}}_{q}, \underline{\mathrm{y}}_{q}\right]=\left[T_{q, p} \underline{\mathrm{x}}_{q}, T_{q, p} \underline{\mathrm{y}}_{q}\right] \text {. }
$$

Thus we have (i) and (ii).

\section{Surjectivity}

Let $\Gamma$ be a calibration for $(E, F)$ and $A \subset E \times F$ be a multivalued map. The range of $A$ will be denoted by $R(A)$.

(3.1). Assume that

(i) $E$ is complete,

(ii) $A_{p}^{-1}$ is a single-valued map for every $p \in \Gamma$,

(iii) $R\left(A_{p}\right)=E[p]$ for every $p \in \Gamma$.

Then $R(\bar{A})=F$.

Proof. Let $y \in E$. Then, by (iii), there exists $\underline{x}_{p} \in D\left(A_{p}\right)$ such that $\left[\mathrm{x}, s_{p}(y)\right] \in A_{p}$ for every $p \in \Gamma$. Assume that $q \geq p$ in $\Gamma$. Then, by (2.3), 


$$
T_{q, p} A_{q=q} \subset A_{p}{ }_{q, p} \stackrel{\mathrm{x}}{=} q
$$

and, since $S_{q}(y) \in A_{q} \underline{x}_{q}$,

$$
S_{p}(y)=T_{q, p} \circ S_{q}(y) \in A_{p} T_{q, p} \underline{\mathrm{x}}_{q},
$$

or $\left[T_{q, p} \underline{=}, S_{p}(y)\right] \in A_{p}$. Then, by $(i i)$, we have $T_{q, p} \underline{\underline{x}}=\underline{\underline{x}}=$.

Therefore, by $(i)$ and (1.1), there exists $x \in E$ such that $S_{p}(x)=\underline{x}$ for all $p \in \Gamma$. Hence, by (2.1), $[x, y] \in \bar{A}$, or $y \in R(\bar{A})$.

The converse of (3.1) is given by the following.

(3.2). Assume that

(i) $R(A)=F$,

(ii) if $\left[x_{i}, y_{i}\right] \in A$ and $\left(y_{i}\right)$ is a p-Cauchy sequence for some $p \in \Gamma$, then $\left(x_{i}\right)$ is also $p$-Cauchy.

Then $R\left(A_{p}\right)=F[p]$.

Proof. Let $\underline{\mathrm{y}} \in F[p]$ and $\left(y_{i}\right) \in \underline{\underline{y}}$. Then, by $(i)$, we can choose $x_{i} \in D(A)$ such that $\left[x_{i}, y_{i}\right] \in A$. By $(i i),\left(x_{i}\right)$ is $p$-Cauchy. Hence $\left(x_{i}\right) \in \underline{\underline{x}}$ for some $\underline{\underline{x}} \in E[p]$. Then it is the definition of $A_{p}$ that $[\underline{\underline{x}}, \underline{\underline{\mathrm{y}}}] \in A_{p}$.

\section{P-contractions}

Let $\Gamma$ be a calibration for $E$. Then a map $f$ of a subset $D(f)$ of $E$ into $E$ is said to be a I-contraction if

$$
p(f(x)-f(y)) \leq p(x-y)
$$

for all $p \in \Gamma$ and $x, y \in D(f)$. When $f$ is a linear map, it is a $\Gamma$-contraction if and only if $p(f(x)) \leq p(x)$ for all $p \in \Gamma$ and $x \in D(f)$. In this case, the following theorem of Moore [4] is of fundamental importance.

(4.1). Let $E$ be a locally convex space and $S$ be an algebraic semigroup of continuous linear maps of $E$ into $E$. Then $S$ is equicontinuous if and only if there is a calibration $\Gamma$ for $E$ such that $S$ 
consists of $\Gamma$-contractions.

From this theorem we can immediately obtain a sufficient condition for a nonlinear map $f$ to be a $\Gamma$-contraction for some $\Gamma$. We recall that $f$ is said to be Gâteaux-differentiable on $D(f)$ if, for each $a \in D(f)$ and $x \in E$, the limit

$$
\lim _{\varepsilon \rightarrow 0} \varepsilon^{-1}(f(a+\varepsilon x)-f(a))=f^{\prime}(a)(x)
$$

exists and $f^{\prime}(a)$ is a continuous linear map on $E$.

(4.2). Let $f$ be a Gâteaux-differentiable map on a convex subset $D(f)$ into $E$. If the set $\left\{f^{\prime}(x): x \in D(f)\right\}$ is contained in an equicontinuous semigroup, there exists a calibration $\Gamma$ for $E$ such that $f$ is a $\Gamma$-contraction.

The proof is an immediate consequence of the mean-value theorem (see [5], p. 15).

When $f$ is a $\Gamma$-contraction and $p \in \Gamma,\left(f\left(x_{i}\right)\right)$ is a $p$-Cauchy sequence whenever $\left(x_{i}\right)$ is a $p$-Cauchy sequence. Hence, for every $\underline{\underline{x}} \in \overline{S_{p}(D(f))}$, we can set

$$
f_{p}(\underline{\underline{x}})=\lim _{i \rightarrow \infty} S_{p}\left(f\left(x_{i}\right)\right)
$$

Then $f_{p}$ is a contraction of $\overline{S_{p}(D(f))}$ into $E[p]$ and

$$
f_{p} \circ S_{p}=S_{p} \circ f
$$

We shall use the following fact later.

(4.3). Let $f$ be a $\Gamma$-contraction and $p \in \Gamma$. If $x_{i} \in D(f)$ and $S_{p}\left(x_{i}\right) \rightarrow \underline{\underline{x}}$ for some $\underline{\underline{x}} \in E[p]$, then $S_{p}\left(f\left(x_{i}\right)\right) \rightarrow f_{p}(\underline{\underline{x}})$.

\section{T-accretive maps}

Let $\Gamma$ be a calibration for $E$. We shall also denote by $\Gamma$ the calibration for $(E, E)$ with the identical components.

A map $A \subset E \times E$ is said to be $\Gamma$-accretive if, for every $\lambda>0$, $(1+\lambda A)^{-1}$ is a single-valued $\Gamma$-contraction. If, furthermore, 
$R(1+\lambda A)=E$, then $A$ is said to be $m-\Gamma$-accretive.

(5.1). For any map $A \subset E \times E$ and $\lambda>0$,

(i) $(1+\lambda A)_{p}=1+\lambda A_{p}$ for all $p \in \Gamma$,

(ii) $\left((1+\lambda A)^{-1}\right)_{p}=(1+\lambda A)^{-1}$ for $a l l \quad p \in \Gamma$.

Proof. If $[\underline{\underline{x}}, \underline{\underline{y}}] \in(1+\lambda A)_{p}$, we can choose $\left[x_{i}, y_{i}\right] \in I+\lambda A$ such that $S_{p}\left(\left[x_{i}, y_{i}\right]\right) \rightarrow[\underline{\underline{x}}, \underline{\underline{y}}]$. Then $\left[x_{i}, \lambda^{-1}\left(y_{i}-x_{i}\right)\right] \in A$ and

$$
S_{p}\left(\left[x_{i}, \lambda^{-1}\left(y_{i}-x_{i}\right)\right]\right) \rightarrow\left[\underline{\underline{x}}, \lambda^{-1}(\underline{\underline{y}-\underline{\underline{x}})}]\right. \text {. }
$$

Hence $\left[\underline{\underline{x}}, \lambda^{-1}(\underline{\underline{y}-\underline{x}})\right] \in A_{p}$ which implies $[\underline{\underline{x}}, \underline{\underline{y}}] \in 1+\lambda A_{p}$. The converse and $(i i)$ can be proved similarly.

We are going to express the $\Gamma$-accretiveness of $A$ in terms of the accretiveness of the family $\left\{A_{p}: p \in \Gamma\right\}$ of maps on Banach spaces.

(5.2). $A$ is $\Gamma$-accretive if and only if every $A_{p}$ is accretive.

Proof. Assume that $A$ is $\Gamma$-accretive and $p \in \Gamma$. Then, for $J_{\lambda}=(1+\lambda A)^{-1}$, we have

$$
p\left(J \lambda^{x-J} \lambda^{y}\right) \leq p(x-y) \text { for } x, y \in R(1+\lambda A) .
$$

Let $\underline{\underline{x}}, \underline{\underline{y}} \in R\left(1+\lambda A_{p}\right)$. Then, by (5.I) (i), there exist $x_{i}, y_{i} \in R(1+\lambda A)$ such that

$$
S_{p}\left(x_{i}\right) \rightarrow \underline{\underline{x}} \text { and } S_{p}\left(y_{i}\right) \rightarrow \underline{\underline{y}}
$$

Then, by (4.3), we have

$$
S_{p}\left(J_{\lambda} x_{i}\right) \rightarrow\left(J_{\lambda}\right)_{p}(\underline{\underline{x}}) \text { and } S_{p}\left(J_{\lambda} y_{i}\right) \rightarrow\left(J_{\lambda}\right)_{p}(\underline{\underline{y}}) .
$$

Hence

$$
\begin{aligned}
p\left(\left(J_{\lambda}\right)_{p}(\underline{\underline{x}})-\left(J_{\lambda}\right)_{p}(\underline{\underline{y}})\right) & =\lim _{i \rightarrow \infty} p\left(J_{\lambda} x_{i}-J_{\lambda} y_{i}\right) \\
& \leq \lim _{i \rightarrow \infty} p\left(x_{i}-y_{i}\right)=p(\underline{\underline{x}}-\underline{\underline{y}})
\end{aligned}
$$


Since $\left(J_{\lambda}\right)_{p}=\left(J_{p}\right)_{\lambda}$ by $(5.1)(i i)$, we have proved that $A_{p}$ is accretive. Conversely, assume that every $A_{p}$ is accretive and $x, y \in R(1+\lambda A)$. Then, for every $p \in \Gamma, S_{p}(x), S_{p}(y) \in R\left(\lambda+\lambda A_{p}\right)$. Therefore,

$$
p\left(\left(J_{p}\right)_{\lambda} S_{p}(x)-\left(J_{p}\right)_{\lambda} S_{p}(y)\right) \leq p(x-y) \text {. }
$$

However, we always have $\left(J_{p}\right)_{\lambda} \circ S_{p}=S_{p} \circ J$. Hence

$$
p\left(J_{\lambda} x-J \lambda^{y}\right) \leq p(x-y)
$$

which shows that $A$ is $\Gamma$-accretive.

(5.3). (i) If $A$ is m-Г-accretive, every $A_{p}$ is m-accretive.

(ii) If $E$ is complete, $A$ is closed and every $A_{p}$ is m-accretive, then $A$ is $m$-Г-accretive.

Proof. (i) By the assumption, $R(1+\lambda A)=E$. To prove that the condition (3.2) ( $i i)$ is satisfied for $1+\lambda A$, assume that

$\left[x_{i}, y_{i}\right] \in I+\lambda A$ and $\left(y_{i}\right)$ is $p$-Cauchy. Then, since $x_{i}=J_{\lambda} y_{i}$ and $J_{\lambda}$ is a $\Gamma$-contraction, $\left(x_{i}\right)$ is also p-Cauchy. Hence, by (3.2), we have $R\left(1+\lambda A_{p}\right)=E[p]$.

(ii) We prove that the conditions (i), (ii) and (iii) in (3.1) are satisfied for $1+\lambda A$. However $(i)$ is a part of our assumptions and (ii) is included in the definition of $A_{p}$ being accretive. Finally, (iii) is satisfied by $(1+\lambda A)_{p}$. Thus we have $R(\overline{1+\lambda A})=E$. However, $\overline{1+\lambda A}=1+\lambda \bar{A}$ and, since $A$ is closed, we arrive at $R(1+\lambda A)=E$.

The following facts are immediate consequences of the definition of $m-\Gamma$-accretiveness and the fact that $m$ - $\Gamma$-accretive maps are "maximal" T-accretive.

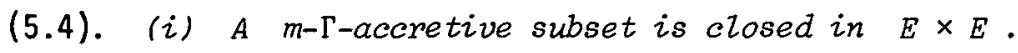

(ii) If $A$ is $m-\Gamma$-accretive and $x \in D(A)$, then $A x$ is closed.

\section{Some function spaces}

Let $E$ be a locally convex space equipped with a calibration $\Gamma$. 
For a positive number $T$, we shall denote the closed interval $[0, T]$ by $I$, and let $C(I, E)$ be the set of all continuous functions of $I$ into $E$. Then, for each $p \in \Gamma$ and $u \in C(I, E), p \circ u$ is a real-valued continuous function defined on the compact subset $I$. Therefore, we can set

$$
p_{t}(u)=\sup \{p(u(s)): 0 \leq s \leq t\}
$$

for $t \in I$, and we set $p^{\infty}(u)=p_{T}^{\infty}(u)$ for $u \in C(I, E)$. Then the set

$$
\Gamma^{\infty}=\left\{p^{\infty}: p \in \Gamma\right\}
$$

defines a locally convex topology on $C(I, E)$ and it is complete if $E$ is complete.

Let $A$ be a multi-valued map on $E$ and we denote by $C(I, \overline{D(A)})$ the set of all continuous functions of $I$ into $\overline{D(A)}$. Then it is a closed subset of $C(I, E)$.

We shall denote the p-completion of $C(I, E)$ by $C(I, E)[p]$. Then we have the natural embedding

$$
S_{p}: C(I, E) \rightarrow C(I, E)[p] .
$$

We now set

$$
C(I, \overline{D(A)})[p]=\overline{S_{p}(C(I, \overline{D(A)}))} .
$$

(6.1). If $\overline{D(A)}$ is convex, $\left.C(I, \overline{D(A)})[p]=C\left(I, \overline{D\left(A_{p}\right.}\right)\right]$.

Proof. By (2.2) (ii), we have

$$
\left.S_{p}(C(I, \overline{D(A)})) \subset C\left(I, \overline{D\left(A_{p}\right.}\right)\right)
$$

and $C\left(I, \overline{D\left(A_{p}\right)}\right)$ is closed. Hence we only need to show that $C\left(I, \overline{D\left(A_{p}\right]}\right] \subset C(I, \overline{D(A)})[p]$. Now let $\left.\underline{\underline{\mathrm{v}}} \in C\left(I, \overline{D\left(A_{p}\right.}\right)\right]$ and we set

$$
\underline{v}_{n}(t)=\frac{k T-n t}{k T} \stackrel{a}{k-1}_{k T}+\frac{n t}{k T} \stackrel{q}{z} k_{\text {if }} \frac{(k-1) T}{n} \leq t \leq \frac{k T}{n}
$$

for $k=1,2, \ldots, n$, where

$$
\mathrm{a}_{k}=\underline{\underline{v}}(k T / n) \text { for } k=0,1, \ldots, n \text {. }
$$


Then, since $\overline{D\left(A_{p}\right)}$ is convex, $\underset{n}{\underline{\mathrm{v}}} \in C\left(I, \overline{D\left(A_{p}\right)}\right)$ and $\underset{n \rightarrow \infty}{\lim } \stackrel{\mathrm{v}}{=}=\underline{\underline{\mathrm{v}}}$ in the Banach space $C(I, E[p])$. Since $\underline{a}_{k} \in \overline{D\left[A_{p}\right]}$, we can choose $a_{k, i} \in D(A)$ such that $s_{p}\left(a_{k, i}\right) \rightarrow \underline{a}_{k}$ for $k=0,1, \ldots, n$, and we define functions $v_{n, i}: I \rightarrow E$ by

$$
v_{n, i}(t)=\frac{k T-n t}{k T} a_{k-1, i}+\frac{n t}{k T} a_{k, i} \text { if } \frac{(k-1) T}{n} \leq t \leq \frac{k T}{n}
$$

Then, since $\overline{D(A)}$ is convex, $v_{n, i} \in C(I, \overline{D(A)})$ and it is easy to see that

$$
p^{\infty}\left(S_{p} \circ v_{n, i}-\stackrel{\mathrm{v}}{=}\right) \rightarrow 0 \text { as } i \rightarrow \infty .
$$

This means that $\left(v_{n, i}\right)$ is a $p$-Cauchy sequence in $C(I, \overline{D(A)})$ and $\left(v_{n, i}\right) \in \underset{\mathrm{v}}{\Rightarrow}$. Hence $\underset{\mathrm{v}_{n}}{\mathrm{v}} \in C\left(I, \overline{\left.D_{1}^{\prime} A\right)}\right)[p]$. Since $C(I, \overline{D(A)})[p]$ is closed, $\underline{\underline{v}} \in C(I, \overline{D(A)})[p]$.

We note that, when $A$ is an m-accretive map on a Banach space which, together with its dual, is uniformly convex, then $\overline{D(A)}$ is conves. (See Barbu [1], p. 77, Proposition 3.6.) We shall call a calibration $\Gamma$ dualty uniformly convex if, for every $p \in \Gamma, E[p]$ and its dual are uniformly convex. Obviously $L_{\text {loc }}^{p}(R)$ admits such a calibration if $1<p<\infty$. It is also known that every nuclear space admits a Hilbert calibration which is obviously dually uniformly convex.

Let, as before, $E$ be a locally convex space equipped with a calibration $\Gamma$, and let $L^{1}(I, E)$ be the set of all integrable functions of $I$ into $E$. Then, for each $p \in \Gamma$ and $u \in L^{1}(I, E)$, we can set

$$
p_{t}^{I}(u)=\int_{0}^{t} p(u(s)) d s \text { for } t \in I \text {, }
$$

and we also set $p^{I}(u)=p_{T}^{I}(u)$. Then the set

$$
\Gamma^{1}=\left\{p^{1}: p \in \Gamma\right\}
$$

defines a locally convex topology on $L^{1}(I, E)$ and it is complete if $E$ is complete. In the same manner as in the case of $C(I, \overline{D(A)})$, we can 
prove that

$$
L^{I}(I, E)[p]=L^{1}(I, E[p]) \text { for every } p \in \Gamma
$$

In the proof of $L^{l}(I, E[p]) \subset L^{\perp}(I, E)[p]$ we can approximate elements of $L^{l}(I, E[p])$ by step functions which are, as can be proved easily, contained in $L^{1}(I, E)[p]$.

\section{A result of Crandall and Nohel}

Let $E$ be a Banach space and $A \subset E \times E$ be an m-accretive map. For $a \in \overline{D(A)}$, let us consider the following initial value problem:

$$
\left\{\begin{array}{l}
\frac{d}{d t} u(t)+A u(t) \rightarrow G u(t) \quad(t \in I), \\
u(0)=a,
\end{array}\right.
$$

where

$$
G: C(I, \overline{D(A)}) \rightarrow L^{\perp}(I, E) \text {. }
$$

It has been proved in [2] that the problem (*) has a unique solution in $C(I, \overline{D(A)})$ if there exists $\gamma \in L^{1}(I, R)$ such that

$$
p_{t}^{I}(G(u)-G(v)) \leq \int_{0}^{t} \gamma(s) p_{s}^{\infty}(u-v) d s
$$

for all $u, v \in C(I, \overline{D(A)})$ and $t \in I$, where $p$ denotes the norm of $E$.

(7.1). Let $E$ be a complete locally convex space equipped with a dually uniformly convex calibration $\Gamma$. If $A \subset E \times E$ is an $m-\Gamma$ accretive map and the map $G$ satisfies the condition (**) for every $p \in \Gamma$, then, for each $a \in \overline{D(A)}$, the initial value problem (*) has a unique solution in $C(I, \overline{D(A)})$.

Proof. The condition (**) implies

$$
p^{I}(G(u)-G(v)) \leq\left(\int_{0}^{T} \gamma(s) d s\right) p^{\infty}(u-v)
$$

for $u, v \in C(I, \overline{D(A)}]$. Hence $\left(G\left(u_{i}\right)\right)$ is $p^{I}$-Cauchy whenever $\left(u_{i}\right)$ is $p^{\infty}$-Cauchy. Therefore, by (6.1), we can define a map 


$$
\left.G_{p}: C\left(I, \overline{D\left(A_{p}\right.}\right)\right] \rightarrow L^{1}(I, E[p])
$$

such that

$$
G_{p}\left(S_{p} \circ u\right)=S_{p} \circ G(u) \text { for every } u \in C(I, \overline{D(A)})
$$

and, if $q \geq p$ in $\Gamma$,

$$
T_{q, p}\left(G_{q}\left(\underline{u}_{q}\right)(t)\right)=G_{p}\left(T_{q, p} \circ \stackrel{\circ}{=}\right)(t)
$$

for all $\underline{\underline{u}}_{q} \in C\left(I, \bar{D}\left(A_{p}\right)\right]$ and $t \in I$. Furthermore, $G_{p}$ satisfies the condition $(* *)$. Hence, for each $p \in \Gamma$, the initial value problem

$$
\left\{\begin{array}{l}
\frac{d}{d t} \underline{\underline{u}}(t)+A_{p} \underline{\underline{u}}(t) \rightarrow G_{p}(\underline{\underline{u}})(t) \quad(t \in I) \\
\underline{\underline{u}}(0)=S_{p}(a)
\end{array}\right.
$$

has a unique solution $\stackrel{\mathrm{u}}{=}$ in $C\left(I, \overline{D\left(A_{p}\right)}\right)$. Then, if $q \geq p$ in $\Gamma$,

$$
T_{q, p} \underline{u}_{q}(0)=T_{q, p}\left(S_{q}(a)\right)=S_{p}(a) \text {, }
$$

and

$$
T_{q, p}\left(\frac{d}{d t} \underline{\underline{u}}_{q}(t)\right)+T_{q, p}\left(A_{q} \underline{u}_{q}(t)\right) \rightarrow T_{q, p}\left(G_{q}\left(\underline{u}_{q}\right)(t)\right)
$$

which, by (2.3), implies

$$
\frac{d}{d t} T_{q, p} \stackrel{u}{=}_{q}(t)+A_{p} T_{q, p} \underline{u}_{q}(t) \rightarrow G_{p}\left(T_{q, p} \circ \underline{\underline{u}}_{q}\right)(t) \quad(t \in I) .
$$

Hence, by the unicity of the solution,

$$
T_{q, p} \underline{u}_{q}(t)=\underline{u}_{p}(t) \text { for all } t \in I \text {. }
$$

Since $E$ is complete, we can apply (1.1) to find $u(t) \in E$ such that

$$
\underline{\underline{u}} p(t)=S_{p}(u(t)) \text { for all } p \in \Gamma \text { and } t \in I \text {. }
$$

Then $u(t)$ is continuous with respect to $t$ and, by (2.2),

$$
u(t) \in \prod_{p \in \Gamma} S_{p}^{-I}\left(\bar{D}\left(A_{p}\right)\right)=\overline{D(A)}
$$

Hence $u \in C(I, \overline{D(A)})$ and, furthermore,

$$
S_{p}\left\{\frac{d}{d t} u(t)+A u(t)\right) \rightarrow S_{p}(G(u)(t)) \text { for all } t \in I \text { and } p \in \Gamma \text {. }
$$


Then, by the lemma which will be proved below, we have

$$
\frac{d}{d t} u(t)+A u(t) \rightarrow G(u)(t) \text { for all } t \in I \text {. }
$$

The lemma referred to in the above is the following.

(7.2). Assume that $B$ is a closed subset of $E$ and

$$
S_{p}(x) \in S_{p}(B) \text { for every } p \in \Gamma \text {. }
$$

Then $x \in B$.

Proof. For each $p \in \Gamma$ we choose $b_{p} \in B$ such that $s_{p}(x)=S_{p}\left(b_{p}\right)$, which means that $p\left(x-b_{p}\right)=0$. Then the net $\left(b_{p}\right)$ converges to $x$. Since $B$ is closed, we have $x \in B$.

\section{References}

[1] Viorel Barbu, Nonlinear semigroups and differential equations in Banach spaces (Noordhoff, Leyden, The Netherlands, 1976).

[2] M.G. Crandall and J.A. Nohel, "An abstract functional differential equation and a related nonlinear Volterra equation", Israel $\mathrm{J}$. Math. 29 (1978), 313-328.

[3] G. Köthe, Topological vector spaces I (Die Grundlehren der mathematischen Wissenschaften, 159. Springer-Verlag, Berlin, Heidelberg, New York, 1967).

[4] Robert T. Moore, "Banach algebras of operators in locally convex spaces", Bull. Amer. Math. Soc. 75 (1969), 68-73.

[5] Sadayuki Yamamuro, Differential calculus in topological linear spaces (Lecture Notes in Mathematics, 374. Springer-Verlag, Berlin, Heidelberg, New York, 1974).

[6] Sadayuki Yamamuro, A theory of differentiation in locally convex spaces (Memoirs of the American Mathematical Society, 212. American Mathematical Society, Providence, Rhode Island, 1979).

[7] Sadayuki Yamamuro, "Notes on the inverse mapping theorem in locally convex spaces", Bull. Austral. Math. Soc. 21 (1980), 419-461. 
[8] Sadayuki Yamamuro, "On the surjectivity of linear maps on locally convex spaces", J. Austral. Math. Soc. Ser. A 32 (1982), 187-211.

Department of Mathematics, Institute of Advanced Studies, Australian National University, PO Box 4, Canberra, ACT 2600, Australia. 